\title{
Editorial: Emerging Cellulosic Materials: Characterization of Structure and Properties
}

\author{
Tiina Nypelö ${ }^{1}$, Per Stenius ${ }^{2 *}$ and Tekla Tammelin ${ }^{3}$ \\ ${ }^{1}$ Department of Chemistry and Chemical Engineering, Chalmers University of Technology, Gothenburg, Sweden, ${ }^{2}$ Centralvägen, \\ Åkersberga, Sweden, ${ }^{3}$ TTT Technical Research Centre of Finland Ltd., Espoo, Finland
}

Keywords: cellulose, nanocellulose, foams, emulsions, films, surface area, 3D printing

\section{Editorial on the Research Topic}

\section{Emerging Cellulosic Materials: Characterization of Structure and Properties}

\section{OPEN ACCESS}

Edited by:

Martino Di Serio,

University of Naples Federico II, Italy

Reviewed by:

María Cristina Area,

Institute of Materials of Misiones

(IMAM), Argentina

*Correspondence:

Per Stenius

perj.stenius@gmail.com

Specialty section:

This article was submitted to Chemical Reaction Engineering,

a section of the journal

Frontiers in Chemical Engineering

Received: 12 January 2022

Accepted: 31 January 2022

Published: 01 March 2022

Citation:

Nypelö T, Stenius $P$ and Tammelin T (2022) Editorial: Emerging Cellulosic Materials: Characterization of Structure and Properties.

Front. Chem. Eng. 4:853268. doi: 10.3389/fCeng.2022.853268
This Research Topic of Frontiers in Chemical Engineering presents research inspired by factors that have become increasingly important during the last decades. Firstly, there is an urgent demand for new and ecologically viable materials that are not based only on non-renewable resources. Secondly, the sustainable utilization of biobased renewables must be fully vindicated and in this, novel value-added products play a key role. Both factors have resulted in research on and development of a plethora of new materials based on three of the most abundant polymers produced by nature and available in forest biomass: cellulose, lignins, and hemicelluloses, for use in a wide variety of applications. So far, most materials have been prepared only in the laboratory, but many are already produced in pilot plant scale or are even manufactured commercially.

The majority of the methods of preparation and intended applications of these materials involve the assembly of particles of nano- and micro scale with vast specific surfaces. Among these, the material of predominant importance is nanocellulose, i.e., cellulose nanocrystals (CNC), cellulose nanofibers (CNF), and bacterial nanocellulose (BC). The contributions in this Research Topic highlight the wide variety of possible uses of nanocellulose in new materials: formation of networks with other biopolymers. (Lohtander et al.; Wang et al.), stabilization of emulsions and foams (Gestranius et al.; Nypelö et al.). The contributions also stress the importance of extending beyond using routine methods to characterize nanocellulose for use in materials formed by nanoscale particles by, for example, adequate and reliable determination of specific surfaces (Kondor et al.), film thickness and structure (Spirk et al.).

The following is a summary of significant results and advances in each contribution.

Nanocellulose is an excellent network former. Lohtander et al. in the contribution Bioactive films from willow bark extract and nanocellulose double network hydrogels report on the combination of a nanocellulose network with a biobased polyphenolic compound to form material comprising of a double network. The contribution advances the field of functionalization of nanocellulose materials but also to use nontoxic biobased components to do so, which is of great importance to advance the utilization and intrinsic benefits of renewable raw materials. The emerging materials are likely to be used for packaging and in pharmaceuticals.

The ability of nanocellulose to form networks with photo-crosslinkable biopolymers is highlighted in the contribution of Wang et al. Rheological and printability assessments on biomaterial Inks of nanocellulose/photo-crosslinkable biopolymer in light-aided 3D Printing. The biopolymer networks were targeted for 3D printing, which is an emerging use in the field of natural polymers, among them, nanocellulose. It requires a meticulous analytical approach to assess 
printability, where it is essential to quantify injectability and rheological properties such as thixotropy.

Nanocelluloses are excellent stabilizers of oil-in-water emulsions. In their contribution Creaming layers of nanocellulose stabilized water-based polystyrene: High-solids emulsions for $3 D$ printing, Gestranius et al. show how the ability of nanocellulose to stabilize interfaces can be extended. Combining Pickering type of stabilization, network formation, and co-emulsifier, enables the preparation of polymer droplets. The encapsulation of the polymer results in creaming that is used for removal of water and hence generation of high solid dope. Here as well, the emerging use of nanocellulose in $3 \mathrm{D}$ printing is in focus. Indeed, the mastering of the colloidal and interfacial phenomena leads to a $3 \mathrm{D}$ printable material that can further be dried while retaining its shape.

Nanocelluloses are also able to stabilize aqueous foams, providing that another amphiphilic component is present to assist the foam formation. The synergistic action of nanocellulose and amphiphilic polymer with a Pickering-type stabilization mechanism described in Gestranius et al. is also presented in the contribution by Nypelö et al., $\mathrm{N}_{2} \mathrm{O}$-assisted siphon foaming of modified galactoglucomannas with cellulose nanofibers. The approach involves nanocellulose and etherified GGM - both wood-sourced raw materials - and results in a selfstanding foam structure. This opens application space for preparing green and lightweight composites. This systematic work clearly demonstrates how the control over interfacial activity is a prerequisite for the formation of desired structures.

The procedures to prepare representative samples and their characterization are equally important aspects when considering the efficient utilization of nanocellulose in novel application fields. Especially, the surface area determination of nanocellulose is far from being trivial. Kondor et al. critically discuss the results achieved using bacterial cellulose in their contribution On the BET surface area of nanocellulose determined using volumetric, gravimetric, and chromatographic adsorption methods. Their conclusions are based on comprehensive theoretical considerations, resulting in an appropriate perception of the surface area data interpretation. In particular, they address surface area assessment as a tool to rank different nanocelluloses provided the measurements are carried out under comparable conditions (temperature, pressure, similar sample preparation).

Spirk et al. follow the same lines of non-trivial characterization of nanocellulose materials. They address the characterization of a nanocellulose-based 2D material family - futuristic nanoarchitectures from renewables for optics and electronics. The paper Current opportunities and challenges in biopolymer thin film analysis - Determination of film thickness reviews the commonly employed surface sensitive techniques, simultaneously considering the well-established but demanding features of biobased materials, i.e., heterogeneity and humidityinduced structural changes. The authors adequately emphasize the necessity to exploit complementing methods, such as AFM, along with indirect methods to accurately assess sub-micron film thickness.

\section{AUTHOR CONTRIBUTIONS}

All authors listed have made a substantial, direct, and intellectual contribution to the work and approved it for publication.

Conflict of Interest: Author TT is employed by VTT Technical Research Centre of Finland Ltd.

The remaining authors declare that the research was conducted in the absence of any commercial or financial relationships that could be construed as a potential conflict of interest.

Publisher's Note: All claims expressed in this article are solely those of the authors and do not necessarily represent those of their affiliated organizations, or those of the publisher, the editors and the reviewers. Any product that may be evaluated in this article, or claim that may be made by its manufacturer, is not guaranteed or endorsed by the publisher.

Copyright $\odot 2022$ Nypelö, Stenius and Tammelin. This is an open-access article distributed under the terms of the Creative Commons Attribution License (CC BY). The use, distribution or reproduction in other forums is permitted, provided the original author(s) and the copyright owner(s) are credited and that the original publication in this journal is cited, in accordance with accepted academic practice. No use, distribution or reproduction is permitted which does not comply with these terms. 